\title{
Age-related differences in simultaneous interarm blood pressure measurements
}

\author{
Martin D. Fotherby, Barnabas Panayiotou and John F. Potter \\ University Department of Medicine for the Elderly, Leicester General Hospital, Leicester LE5 4PW, UK
}

\begin{abstract}
Summary: Simultaneous noninvasive blood pressure measurements were recorded bilaterally in 40 young and 40 elderly subjects. Overall interarm blood pressure (BP) differences for the elderly and young groups were similar, the absolute interarm differences being for systolic blood pressure (SBP) elderly: $4.2 \mathrm{mmHg}$ (95\% CI 3.1 -5.3 mmHg); young $3.3 \mathrm{mmHg}$ (2.6-4.1 mmHg); diastolic blood pressure (DBP) elderly $3.6 \mathrm{mmHg}(2.8-4.4 \mathrm{mmHg})$, young $2.7 \mathrm{mmHg}(2.0-3.3 \mathrm{mmHg})$. However, the range of interarm BP differences was wide. Four (10\%) of the elderly had an interarm SBP difference $>10 \mathrm{mmHg}$ compared to one (3\%) of the young group. Interarm DBP differences $>8 \mathrm{mmHg}$ were found in three $(8 \%)$ of the elderly and in none of the young group. Although age does not affect mean interarm BP differences, clinically important interarm BP differences exist in both young and elderly subjects. Blood pressure should be measured in both arms of all patients at initial assessment to avoid potential problems with misclassification of blood pressure status.
\end{abstract}

\section{Introduction}

Large blood pressure (BP) differences may exist between arms which could have important consequences when assessing BP levels. ${ }^{1,2}$ As older persons have a greater prevalence of atheromatous vascular disease, this between arm BP discrepancy may be greater in this age group. The aim of the present study was to compare noninvasive simultaneous interarm BP differences between young and elderly subjects.

\section{Methods}

Forty young subjects (mean age 31 years, range $18-48$ years, eight males) with SBP and DBP range $102-153 / 60-95 \mathrm{mmHg}$ and 40 elderly subjects (mean age 74 years, range 63-85 years, 14 male) with a BP range $98-204 / 53-110 \mathrm{mmHg}$, were recruited from hospital in-patients and outpatients. Subjects were in sinus rhythm with no carotid or subclavian bruits. Simultaneous BP measurements were taken in each arm, using two SpaceLabs 90207 BP monitors with subjects seated, both arms relaxed by their side, using cuffs of the appropriate size. Eight readings were taken in each arm with the monitors being swapped over after four simultaneous recordings. The mid-arm circumference of each arm was recorded.

Correspondence: M.D. Fotherby, M.R.C.P.

Accepted: 5 October 1992

\section{Analysis}

For each subject the mean of eight BP readings each arm was derived and then the BP difference between each arm calculated. Interarm BP differences were compared using unpaired Student's $t$-tests with Pearson's correlation coefficient.

\section{Results}

Mean systolic and diastolic blood pressure levels were significantly greater in the elderly than the 3 . young $(P<0.01)$. However, there was no signfi- $\dot{-}$ cant age-related interarm BP difference for the group as a whole (see Table I) whether values wereô expressed as actual differences or related to baseline BP levels. However, the distribution of응 absolute interarm BP differences varied between $>$ the two groups. Nine $(23 \%)$ of the elderly had an interarm SBP difference of greater than $8 \mathrm{mmHg}$ N and four $(10 \%)$ a difference greater than $10 \mathrm{mmHg}$, compared to only one $(3 \%)$ of the young having a greater than $8 \mathrm{mmHg}$ difference. Three of the elderly and none of the young had interarm DBPO differences greater than $8 \mathrm{mmHg}$. When interarmo BP differences are expressed as a percentage of the mean BP, seven $(18 \%)$ of the elderly have an? interarm SBP difference greater than $6 \%$ (equiva- $\square$ lent to approximately $10 \mathrm{mmHg}$ for a mean BP of $160 \mathrm{mmHg}$ ) compared to two $(5 \%)$ in the young; $\stackrel{\mathbb{Q}}{\stackrel{Q}{Q}}$ and a greater than $6 \%$ interarm diastolic $\mathrm{BP} \stackrel{\mathrm{Q}}{\varrho}$ 
Table I Mean blood pressure with standard deviation [ ] below, in each arm for elderly and young subjects and the mean absolute BP difference (calculated by assuming all differences are positive) between arms with $95 \%$ confidence intervals ( ) and below this, the between arm difference expressed as a percentage of the mean blood pressure

\begin{tabular}{lccccclll}
\hline & \multicolumn{3}{c}{$\begin{array}{c}\text { Mean blood pressure } \\
\text { Elderly }\end{array}$} & \multicolumn{2}{c}{ Young } & \multicolumn{2}{c}{ Absolute mean difference (mmHg) } \\
& $R$ arm & $L$ arm & $R$ arm & L arm & & & \multicolumn{1}{c}{ Young } \\
& & & & & & & & \\
\hline SBP & 148.4 & 149.2 & 125.8 & 125.5 & 4.2 & $(3.1-5.3)$ & 3.3 & $(2.6-4.1)$ \\
& {$[21.7]$} & {$[22.3]$} & {$[9.8]$} & {$[10.4]$} & $2.9 \%(2.1-3.6 \%)$ & $2.6 \%(2.1-3.2 \%)$ \\
DBP & 83.9 & 84.6 & 77.7 & 77.8 & 3.6 & $(2.8-4.4)$ & 2.7 & $(2.0-3.3)$ \\
& {$[13.2]$} & {$[13.6]$} & {$[8.1]$} & {$[8.3]$} & $4.4 \%(3.6-5.4 \%)$ & $3.5 \%(2.7-4.3 \%)$ \\
\hline
\end{tabular}

difference for eight $(20 \%)$ of the elderly and seven $(18 \%)$ of the young. There was no significant correlation between mean BP levels and interarm BP differences for either young, old or for the group as a whole. Mean mid-arm circumference was similar in both groups and was not related to interarm BP differences.

\section{Discussion}

We have shown that there are no significant age-related differences in simultaneous interarm BP measurements. Use of simultaneous, rather than sequential BP measurements has the advantage of reducing variations in BP, other than those due to interarm differences; beat-to-beat variations in BP are reduced and the use of automatic BP recorders eliminates observer bias, while changing recorders between arms will reduce any inherent recorder bias. Interarm BP differences of clinical significance do exist in both young and old, highlighting the need for BP measurments in both arms during initial assessment. Other studies which have not considered the effects of age reported smaller interarm differences; Gould et al. ${ }^{1}$ found $16 \%$ of patients (mean age 50 years) had an SBP interarm difference greater than $6 \mathrm{mmHg}$ or more, compared to $23 \%$ of the elderly group having a greater than $8 \mathrm{mmHg}$ difference in the present study.

Harrison et al. ${ }^{2}$ using noninvasive simultaneous recordings found a $10 \mathrm{mmHg}$ interarm difference in $5 \%$ of patients (mean age 48 years) for SBP and $4 \%$ for DBP, compared to $10 \%$ for SBP and 3\% for DBP in our elderly group; they like us, noted no significant correlation between BP levels and interarm differences. These between arm differences with age could not be accounted for by differences in arm circumference.

The causes of true interarm BP differences may involve vessel atheroma, emboli and thrombi which are more prevalent in the elderly and in hypertensives. Interarm SBP differences of greater than $10 \mathrm{mmHg}$ occurring in $10 \%$ of the elderly and $3 \%$ of the young may lead to spurious results in the assessment of BP levels. Similarly the true effects of anti-hypertensive therapy may be inadequately estimated in individual patients if the same arm (that with the highest blood pressure) is not used on each occasion. It is recommended that BP be taken in both arms at least once in all hypertensive patients, ${ }^{3}$ although one survey found only $23 \%$ of physicians carried this out. ${ }^{4}$

Clinically important BP differences may exist between arms in the young and elderly, which if not appreciated may result in underdiagnosis of hypertension. Even a relatively small underestimation of a patient's blood pressure may be of clinical significance; for example, on a population basis a $5 \mathrm{mmHg}$ difference in clinic DBP has been associated with a $34 \%$ difference in stroke risk. ${ }^{5}$ Also for clinical trials in which changes of blood pressure are being recorded, if the same arm of the subject is not used throughout for blood pressure measurement, blood pressure variability may be increased resulting in a reduced power of the study to detect a given blood pressure difference. It is important to appreciate that the difference of $8 \mathrm{mmHg}$ or more we have found between arms in $23 \%$ of the elderly is in the range of blood pressure reduction achieved in trials of non-pharmacological treatments in the elderly. ${ }^{6,7}$ Use of different arms for blood pressure measurement in such trials may in individuals distort the blood pressure changes of a given intervention. We recommend that all patients have their BP measured in both arms on at least one occasion particularly in hypertensive patients and those in the 'borderline hypertensive' range, so that clinically important interarm differences are not missed. 


\section{References}

1. Gould, B.A., Hornung, R.S., Kieso, H.A., Altman, D.G. \& Raftery, E.B. Is the blood pressure the same in both arms? Clin Cardiol 1985, 8: 423-426.

2. Harrison, E.G., Roth, G.M. \& Hines, E.A. Bilateral indirect and direct arterial pressures. Circulation 1960, 22: 419-436.

3. Muller, F.B. \& Laragh, J.H. Clinical evaluation and differential diagnosis of the individual hypertensive patient, In: Laragh, J.H. \& Brenner, B.M. (ed.) Hypertension, Pathophysiology, Diagnosis and Management. Raven Press, New York, 1990, p. 1389.

4. McKay, C.W., Campbell, N.R.C., Parab, L.S., Chockalingam, A. \& Fodor, J.G. Clinical assessment of blood pressure. $J$ Hum Hypertens 1990, 4: 639-645.
5. MacMahon, S., Peto, R., Cutler, J. et al. Blood pressure, $\underset{\mathbb{\$}}{\}$ stroke, and coronary heart disease. Part 1, prolonged differ- $\varrho$ ence in blood pressure: prospective observational studies. corrected for the regression dilution bias. Lancet 1990, 335: $\overrightarrow{\vec{B}}$ $765-774$.

6. The Trials of Hypertension Prevention Collaborative Research Group. The effects of non-pharmacologic interven- $\bar{O}$ tions on blood pressure of persons with high normal levels. Results of the trials of hypertension prevention, Phase 1 JAMA 1992, 267: 1213-1220.

7. Fotherby, M.D. \& Potter, J.F. Potassium supplementation reduces clinic and ambulatory blood pressure in elderly hypertensive subjects. J Hypertens 1992, 10: 1403-1408. 